\title{
Preoperative ventricular late potentials and the risk of adverse events in patients with obstructive hypertrophic cardiomyopathy undergoing septal myectomy
}

\author{
Yang Zhang ${ }^{1}$, Changpeng Song ${ }^{1}$, Changsheng Zhu ${ }^{2}$, Jian Zhang ${ }^{1}$, Xinxin Zheng ${ }^{1}$, Jie Lu ${ }^{1}$, Shuiyun Wang ${ }^{2}$, \\ Xiaohong Huang' \\ ${ }^{1}$ Department of Special Medical Treatment Center, Fuwai Hospital, National Center for Cardiovascular Diseases, Chinese Academy of Medical \\ Sciences and Peking Union Medical College, Beijing, China; ${ }^{2}$ Department of Cardiovascular Surgery, Fuwai Hospital, National Center for \\ Cardiovascular Diseases, Chinese Academy of Medical Sciences and Peking Union Medical College, Beijing, China \\ Contributions: (I) Conception and design: Y Zhang, X Huang; (II) Administrative support: C Song, C Zhu; (III) Provision of study materials or \\ patients: S Wang; (IV) Collection and assembly of data: Y Zhang, J Zhang, X Zheng, J Lu; (V) Data analysis and interpretation: All authors; (VI) \\ Manuscript writing: All authors; (VII) Final approval of manuscript: All authors. \\ Correspondence to: Xiaohong Huang, MD. Department of Special Medical Treatment Center, Fuwai Hospital, National Center for Cardiovascular \\ Diseases, Chinese Academy of Medical Sciences and Peking Union Medical College, No.167 North Lishi Road, Xicheng District, Beijing 100037, \\ China. Email: huangxiaohong@fuwaihospital.org.
}

Background: Ventricular late potentials (VLPs) detected by signal-averaged electrocardiogram are considered as an indicator of electrical instability in diseased myocardium. Little information exists about the VLPs and their effects on clinical prognosis in patients with obstructive hypertrophic cardiomyopathy (OHCM) undergoing septal myectomy.

Methods: Patients with OHCM who underwent septal myectomy from January 2019 to December 2019 were prospectively enrolled. All patients underwent signal-averaged electrocardiogram. Patients were subsequently divided into two groups based on the presence or absence of VLPs. All patients were followed up after surgery to obtain information of survival status and adverse events, including all-cause mortality, congestive heart failure requiring hospitalization, new-onset stroke, and unexplained syncope.

Results: In total, 128 patients $(47.5 \pm 12.8$ years, $57.8 \%$ male) were enrolled. There were $21(16.4 \%)$ individuals in the VLPs-positive group and 107 (83.6\%) individuals in the VLPs-negative group. There were no statistically significant differences in non-sustained ventricular tachycardia and late gadolinium enhancement on cardiovascular magnetic resonance images between the two groups. Eight adverse events were reported at the $14.9 \pm 4.1$ months follow-up. The rates of adverse events in groups of VLPs-positive and VLPs-negative were $23.8 \%(5 / 21)$ and 2.8\% (3/107) of cases, respectively. These events included three patients hospitalized for heart failure, two who experienced ischemic strokes, and three with unexplained syncope, respectively. Patients in the VLPs-positive group had a higher risk of adverse events than those in the VLPs-negative group $(\mathrm{P}<0.001)$. The presence of positive VLPs (hazard ratio $=9.095$, 95\% confidence intervals: $2.080-39.776, \mathrm{P}=0.003$ ) was a strong independent risk factor of adverse events by multivariate Cox regression analysis. Cardiac function Class III or IV, as defined by the New York Heart Association classification, was also an independent risk factor of adverse events (hazard ratio $=13.756,95 \%$ confidence intervals: $1.667-113.510, \mathrm{P}=0.015)$.

Conclusions: VLPs may increase the risk of adverse events in patients with OHCM after septal myectomy, which may be used as a screening test for further risk stratification.

Keywords: Hypertrophic cardiomyopathy (HCM); ventricular late potentials (VLPs); surgery; prognosis

Submitted Feb 10, 2021. Accepted for publication Jun 02, 2021.

doi: 10.21037/apm-21-367

View this article at: https://dx.doi.org/10.21037/apm-21-367 


\section{Introduction}

Hypertrophic cardiomyopathy (HCM) is a common genetic heart disease that affects approximately 1/500-1/200 of the general population (1). About two-thirds of patients present with left ventricular outflow tract (LVOT) obstruction, which is known as obstructive hypertrophic cardiomyopathy (OHCM) (2). Septal myectomy is the standard of treatment used to relieve LVOT obstruction in patients with medically refractory symptoms (3). Most patients can achieve a life span similar to the age- and sex-matched general population after the procedure $(3,4)$.

Previous studies have reported several risk factors associated with postoperative outcomes of patients with OHCM undergoing septal myectomy. Preoperative risk factors include increasing age, elevated levels of preoperative $\mathrm{N}$-terminal pro-brain natriuretic peptide, and left atrial enlargement $(5,6)$. It is important to investigate novel risk factors to identify patients at high risk after surgery so that early intervention can be achieved.

Ventricular late potentials (VLPs) detected by signalaveraged electrocardiography (SAECG) reflect the delayed conduction of electrical activity through the diseased myocardium (7). VLPs are potential anatomical substrates of ventricular arrhythmias, mostly located in areas within or adjacent to the myocardial infarct region, and they have been used as a marker of electrical instability in several diseases, including arrhythmogenic right ventricular cardiomyopathy $(8,9)$. Previous studies have also suggested that VLPs are associated with the prognosis of primary cardiac diseases or other diseases involving the heart (10-12).

VLPs are present in a significant proportion of patients with $\mathrm{HCM}$, with reported incidence rates varying from $20 \%$ to $30 \%(8,10,13)$. VLPs are associated with structural anomaly and delayed conduction of electrical activity in the diseased myocardium which may contribute to adverse events. We hypothesized that the presence of preoperative VLPs would be linked to the prognosis in patients with OHCM after septal myectomy in patients with OHCM. Few studies have explored the relationship between VLPs and the prognosis of patients with HCM (10). Further studies are needed to evaluate the applicative value of VLPs in patients with HCM. Therefore, the purpose of this study was to determine the incidence of VLPs and associated prognosis in patients with OHCM after septal myectomy.

We present the following article in accordance with the STROBE reporting checklist (available at http://dx.doi. org/10.21037/apm-21-367).

\section{Methods}

\section{Participants}

This was a single-center, prospective, observational study. We studied consecutive patients with OHCM who were referred to Fuwai Hospital in Beijing, China from January 2019 to December 2019. All patients underwent septal myectomy. Inclusion criteria were age $\geq 18$ years old and a diagnosis of OHCM. Patients with sustained atrial fibrillation, previous atrial myectomy, history of implantable cardioverter-defibrillator implantation or cardiac resynchronization therapy, complete left bundle branch block or right bundle branch block were excluded. HCM was defined as primary myocardial hypertrophy with a maximum wall thickness of $\geq 15$ or $\geq 13 \mathrm{~mm}$ in patients with a positive family history. In addition, patients with a left ventricular outflow gradient of $\geq 30 \mathrm{mmHg}$ at rest or on provocation were diagnosed with OHCM. Patients with positive VLPs were assigned to the VLPs-positive group. All other patients were assigned into the VLPs-negative group.

The study conformed to the provisions of the Declaration of Helsinki (as revised in 2013). The study was approved by the Ethics Committee of Fuwai Hospital (NO. 2017-887) and informed consent was obtained from all individual participants.

\section{Signal-averaged electrocardiogram measurement}

SAECG (GE Marquette MAC 5500, GE Medical Systems, Milwaukee, WI, USA) was performed in all patients. Subjects were informed to rest for $10 \mathrm{~min}$ prior to examination. About 200 beats were filtered and averaged. The cut-off of frequency was between $40 \mathrm{~Hz}$ and $250 \mathrm{~Hz}$. The basic noise was below $0.5 \mu \mathrm{V}$. Sample data were recorded and then analyzed by an experienced observer. The following indicators were measured and recorded: heart rate; filtered QRS duration (fQRS); the root mean square voltage in the terminal $40 \mathrm{~ms}$ of filtered QRS complex (RMS40, $\mu \mathrm{V})$; and duration of the terminal part of the QRS complex with a low amplitude of $<40 \mu \mathrm{V}$ (LAS40, ms). Abnormal indicators were defined as $\mathrm{fQRS} \geq 114 \mathrm{~ms}$, RMS40 $\leq 20 \mu \mathrm{V}$, and LAS40 $\geq 38 \mathrm{~ms}$. The presence of two or more abnormal indicators was considered to be positive VLPs (14).

\section{Holter electrocardiogram monitoring}

All study subjects underwent 24-hour Holter 
electrocardiogram monitor. Non-sustained ventricular tachycardia (NSVT) was defined as a heart rate of at least 100 beats/min with an episode of three consecutive ventricular beats. The maximum duration of an episode was $<30 \mathrm{~s}$ and the episodes did not include atrial fibrillation. Data were collected by an experienced researcher cardiologist who was blinded to the clinical data and followup events.

\section{Cardiovascular magnetic resonance (CMR) image acquisition and analysis}

CMR was performed using a $1.5 \mathrm{~T}$ magnetic resonance imaging scanner (MAGNETOM Avanto, Siemens, Erlangen, Germany). Images obtained from CMR were analyzed by a single experienced observer who was blinded to all patients. CMR was performed with a cine sequence. Late gadolinium enhancement (LGE) images were collected in the same views as the cine images $10-15$ min after intravenous administration of $0.2 \mathrm{mmol} / \mathrm{kg}$ gadoliniumDTPA with a breath-held phase-sensitive segmented inversion-recovery sequence.

\section{Echocardiography}

All patients underwent a preoperative echocardiogram, including two-dimensional and Doppler type. Data about cardiac function, left ventricular chamber size, wall thickness, and ventricular and valve function were obtained. The diameter of the cardiac chambers was detected as the maximum value of the anteroposterior diameter in cardiac cycles according to the standard protocol at baseline. The thicknesses of the ventricular wall and the interventricular septum were determined at end-diastole. The LVOT gradient was measured using Doppler echocardiography. Left ventricular ejection fraction was determined according to modified recommendations by the American Society of Echocardiography (15).

\section{Follow-up and outcomes}

All patients were followed up after surgery through a phone conversation with them or their family members every 3 months until October 2020. Adverse events included all-cause mortality, congestive heart failure requiring hospitalization, new-onset stroke, and unexplained syncope. All-cause mortality was defined as any cause of death. Congestive heart failure requiring hospitalization was defined as hospitalization for clinical signs or symptoms of heart failure requiring intravenous diuretics (16). Newonset stroke was defined as the first documentation of a new clinical diagnosis of stroke in the patient's medical record (17). Syncope was defined as self-limited loss of consciousness, which was transitory with spontaneous recovery (18).

\section{Statistical analysis}

Continuous variants with normal distributions were expressed as mean \pm standard deviation and were compared using the two-sample Student's $t$-test. Continuous variables with non-normal distributions are expressed as the median (interquartile range) and were compared using the MannWhitney $U$ test. Categorical measures were presented as numbers (percentages) and were compared using Chisquare or Fisher's exact test.

Survival free from the adverse events of this study was calculated by Kaplan-Meier survival analysis, with two groups compared using the log-rank test. Both univariate and multivariate regression analyses were conducted to determine the independent risk factors of adverse events. The Cox regression analysis was used to estimate hazard ratios (HR) and $95 \%$ confidence intervals (CI). The independent risk factors of adverse events were determined using a Cox proportional hazards regression model. Variables with the $\mathrm{P}$ value $<0.5$ in univariate analysis or were considered clinically relevant (age and sex) were included in multivariate analysis. All reported probability values were two-tailed, and a $\mathrm{P}$ value $<0.05$ was considered statistically significant. Statistical analyses were performed using SPSS version 24.0 software (IBM Corporation, Armonk, NY).

\section{Results}

\section{Study population}

Based on the exclusion criteria, 80 patients were excluded from the study (Figure 1). Patient characteristics are presented in Table 1. The present study comprised 128 patients with OHCM who underwent SAECG, of whom $74(57.8 \%)$ were male. The mean age of the patients was $47.5 \pm 12.8$ (range, 19.0-71.0) years old. Positive VLPs were observed in 21 (16.4\%) of 128 patients who underwent SAECG. One, two, or three abnormal indicators recorded by SAECG were noted in 57, 1, and 20 patients, respectively. 


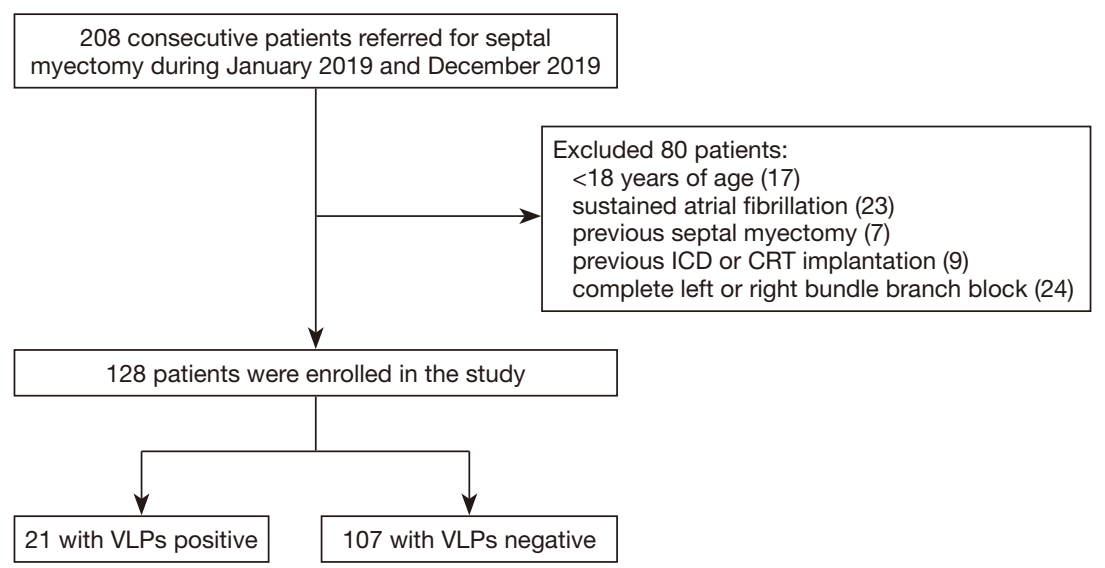

Figure 1 Flowchart of the study population. VLPs, ventricular late potentials; CRT, cardiac resynchronization therapy; ICD, implantable cardioverter-defibrillator.

\section{The important clinical variables in different groups}

The two groups did not differ significantly at baseline regarding demographic or clinical variables. Interventricular septal thickness, left ventricular end-diastolic diameter, and left ventricular posterior wall thickness using echocardiography were higher in the VLPs-positive group than in the VLPs-negative group, although these differences were not statistically significant.

No significant difference was detected regarding NSVT between the groups based on the presence of VLPs $(\mathrm{P}=0.134)$ seen on a $24-\mathrm{h}$ dynamic electrocardiogram.

There were 107 patients who underwent CMR, of whom, 84 of whom presented with LGE on CMR. The presence of LGE did not differ significantly between the two groups $(\mathrm{P}=0.824)$.

\section{Follow-up of adverse events}

The median follow-up time was $14.9 \pm 4.1$ months. During the follow-up period, no patients died. The total number of adverse events was eight: three patients were hospitalized again due to heart failure, two had ischemic strokes, and three experienced unexplained syncope.

Five follow-up adverse events $(23.8 \%, 5 / 21)$ occurred in the VLPs-positive group, while three $(2.8 \%, 3 / 107)$ occurred in the VLPs-negative group. The incidence of total adverse events was higher in the VLPs-positive group than in the VLPs-negative group. Kaplan-Meier curve analysis showed that patients with positive VLPs showed significantly lower event-free rates than those with negative VLPs $(\mathrm{P}<0.001)$ (Figure 2).
Cox multivariate regression analysis showed that the presence of positive VLPs (hazard ratio $=9.095$, 95\% CI: 2.080-39.776, $\mathrm{P}=0.003$ ) was an independent risk factor for adverse events during follow-up (Table 2). Furthermore, New York Heart Association (NYHA) Class III or IV was also found to be an independent risk factor for adverse events (hazard ratio $=13.756,95 \% \mathrm{CI}: 1.667-113.510, \mathrm{P}=0.015$ ).

\section{Discussion}

To the best of our knowledge, this is the first study to demonstrate the clinical characteristics and prognostic significance of VLPs in patients with OHCM after septal myectomy. There were three major findings of the present study. First, we determined the incidence of VLPs in patients with OHCM. Second, VLPs were not related to NSVT or cardiac fibrosis detected by CMR at baseline data. Third, VLPs were significantly associated with adverse events during follow-up when adjusted for age, sex, and cardiac function (as classified by the NYHA classification).

Previous studies have reported that the incidence of VLPs in patients with HCM was $20-30 \%$ in $\operatorname{HCM}(8,10,13)$, which is similar to that reported in our study. The incidence of VLPs in patients with HCM was higher than that in the general population, which suggests that abnormal conduction occurs in diseased cardiac tissues $(8,19)$.

VLPs represent delayed conduction through a diseased myocardium and indicate the presence of potential anatomical features of macro reentry ventricular arrhythmia in several diseases, mostly in patients with acute myocardial infarction (20). Simson et al. and Breithardt et al. were 
Table 1 Baseline characteristics of the studied patients.

\begin{tabular}{|c|c|c|c|c|}
\hline Characteristic & Total $(n=128)$ & VLPs-positive $(n=21)$ & VLPs-negative $(n=107)$ & $\mathrm{P}$ \\
\hline BMI, $\mathrm{kg} / \mathrm{m}^{2}$ & $26.3 \pm 3.6$ & $25.5 \pm 3.5$ & $26.5 \pm 3.6$ & 0.303 \\
\hline Male, n (\%) & $74(57.8)$ & $11(52.4)$ & $63(58.9)$ & 0.581 \\
\hline Course of disease, years (IQR) & $36.0(14.0-108.0)$ & $34.0(7.5-96.0)$ & $39.0(24.0-108.0)$ & 0.503 \\
\hline Hypertension, n (\%) & $31(24.2)$ & 7 (33.3) & $24(22.4)$ & 0.286 \\
\hline CAD, n (\%) & $13(10.2)$ & $2(9.5)$ & $11(10.3)$ & 0.916 \\
\hline \multicolumn{5}{|l|}{ Clinical presentation, n (\%) } \\
\hline Chest pain & $26(20.3)$ & $3(14.3)$ & $23(21.5)$ & 0.453 \\
\hline NSVT, n (\%) & $20(15.6)$ & $1(4.8)$ & $19(17.8)$ & 0.134 \\
\hline LGE, n/total (\%) & 84/107 (78.5) & 13/17 (76.5) & $71 / 90(78.9)$ & 0.824 \\
\hline BNP, pg/mL (IQR) & $935.8(375.6-1,778.0)$ & $1,608.0(834.5-2,114.5)$ & $827.8(365.0-1,733.0)$ & 0.083 \\
\hline \multicolumn{5}{|l|}{ Echocardiographic indices } \\
\hline LAD, mm & $43.6 \pm 5.9$ & $45.5 \pm 7.5$ & $43.3 \pm 5.5$ & 0.337 \\
\hline IVST, mm & $19.2 \pm 4.2$ & $20.6 \pm 4.2$ & $18.9 \pm 5.5$ & 0.111 \\
\hline LVEF, \% & $70.5 \pm 4.7$ & $71.0 \pm 5.7$ & $70.4 \pm 4.6$ & 0.671 \\
\hline LVEDD, mm & $42.6 \pm 4.6$ & $44.1 \pm 4.6$ & $42.3 \pm 4.6$ & 0.123 \\
\hline \multicolumn{5}{|l|}{ SAECG variables } \\
\hline fQRS, ms & $122.9 \pm 19.9$ & $159.0 \pm 17.6$ & $115.8 \pm 10.4$ & $<0.001$ \\
\hline fQRS > 118 ms, n (\%) & $78(60.9)$ & $21(100.0)$ & $57(53.3)$ & $<0.001$ \\
\hline $\mathrm{RMS}, \mu \mathrm{V}$ & $48.5(31.0-72.8)$ & $1.0(1.0-3.0)$ & $52.0(37.0-77.0)$ & $<0.001$ \\
\hline $\mathrm{RMS}<20 \mu \mathrm{V}, \mathrm{n}(\%)$ & $20(15.6)$ & $20(95.3)$ & $0(0)$ & $<0.001$ \\
\hline LAS, ms & $27.0(21.0-34.0)$ & $63.0(52.0-67.0)$ & $25.0(19.0-30.0)$ & $<0.001$ \\
\hline LAS >38 ms, n (\%) & $21(16.4)$ & $21(100.0)$ & $0(0)$ & $<0.001$ \\
\hline
\end{tabular}

BMI, body mass index; BNP, N-terminal pro-brain natriuretic peptide; CAD, coronary artery disease; fQRS, filtered QRS complex duration; IVST, interventricular septal thickness; LAD, left atrium diameter; LAS40, duration of the terminal part of the QRS complex with a low amplitude $<40 \mu \mathrm{V}$; LGE, late gadolinium enhancement; LVEDD, left ventricular end-diastolic diameter; LVEF, left ventricular ejection; LVOT, left ventricular outflow tract; LVPWT, left ventricular posterior wall thickness; MR, mitral regurgitation; NSVT, non-sustained ventricular tachycardia; NYHA, New York Heart Association; RMS40, root mean square voltage in the terminal 40 ms of filtered QRS complex; SAECG, signal-averaged electrocardiogram measurement; VLPS, ventricular late potentials. 
the firsts to demonstrate the clinical value of VLPs in identifying patients with sustained ventricular tachycardia $(21,22)$. Since then, researchers have mostly focused on identifying ventricular arrhythmias after myocardial infarction and then extended their findings to other cardiac

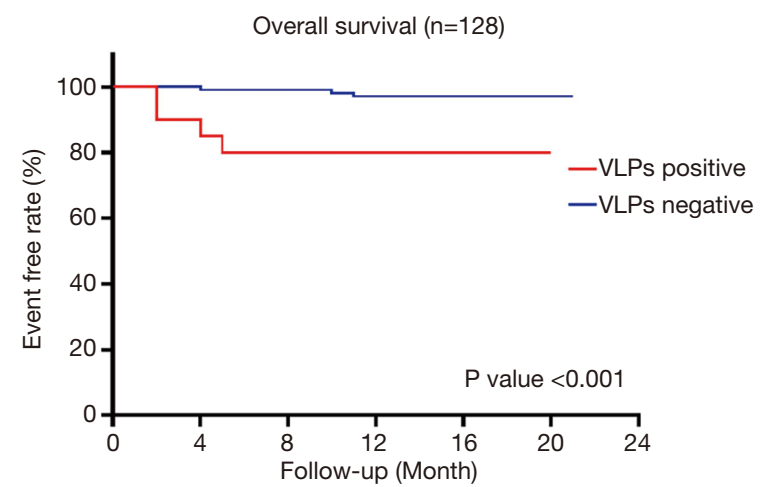

Number at risk

$\begin{array}{ccccccc}\text { VLPs positive } & 20 & 18 & 17 & 16 & 8 & 1 \\ \text { VLPs negative } & 107 & 107 & 106 & 88 & 54 & 12\end{array}$

Figure 2 Kaplan-Meier event-free curves of patients with OHCM. The adverse event rate was significantly higher in VLPs-positive group than in VLPs-negative group $(\mathrm{P}<0.001)$. VLPs, ventricular late potentials; $\mathrm{OHCM}$, obstructive hypertrophic cardiomyopathy. and extracardiac diseases. With the deepening of research, scholars have suggested that VLPs have a low positive predictive value but had a high negative predictive value for ventricular arrhythmic events (7).

In our study, no significant difference was detected regarding the presence of NSVT between the two groups. This implies that SAECG has a limited role in identifying ventricular arrhythmias in patients with OHCM at admission. This result was in line with that of previous studies conducted in patients with HCM. Marstrand et al. reported that SAECG was not always useful for identifying ventricular arrhythmias or sudden cardiac death in patients with HCM (23). It is recommended that investigators combine VLPs with other clinical markers to better identify patients at high risk of ventricular arrhythmias. The relationship between VLPs and ventricular arrhythmias should be further evaluated in larger prospective studies.

Several studies have discussed the correlations between VLPs and cardiac fibrosis. Cardiac fibrosis can be assessed non-invasively by LGE on CMR. It is speculated that increased myocardial fibrosis can lead to positive VLPs, indicating that myocardial fibrosis may be related to delayed conduction of myocardial electrical activity. However, this conclusion cannot be definitively confirmed. Matsuki et al.

Table 2 Univariable and multivariable Cox regression analyses to identify adverse events

\begin{tabular}{|c|c|c|c|c|}
\hline \multirow{2}{*}{ Variable } & \multicolumn{2}{|l|}{ Univariable } & \multicolumn{2}{|l|}{ Multivariate } \\
\hline & $\mathrm{HR}(95 \% \mathrm{Cl})$ & $\mathrm{P}$ & $\mathrm{HR}(95 \% \mathrm{Cl})$ & $P$ \\
\hline Age & $1.049(0.985-1.118)$ & 0.139 & $1.026(0.962-1.095)$ & 0.438 \\
\hline Sex & $4.235(0.858-21.075)$ & 0.076 & $3.581(0.677-18.937)$ & 0.133 \\
\hline Body mass index & 1.160 (0.969-1.389) & 0.106 & & \\
\hline LVEDD & $0.996(0.859-1.156)$ & 0.962 & & \\
\hline IVST & $0.906(0.836-1.171)$ & 0.906 & & \\
\hline LVEF & $1.075(0.932-1.240)$ & 0.322 & & \\
\hline LVOT gradient & $0.987(0.966-1.008)$ & 0.217 & & \\
\hline Mitral valve procedure & $0.743(0.091-6.039)$ & 0.781 & & \\
\hline VLPS & $9.722(2.231-40.731)$ & 0.002 & 9.095 (2.080-39.776) & 0.003 \\
\hline
\end{tabular}

CABG, coronary artery bypass grafting; CAD, coronary artery disease; Cl, confidence intervals; HR, hazard ratio; IVST, interventricular septal thickness; LGE, late gadolinium enhancement; LVEDD, left ventricular end-diastolic diameter; LVEF, left ventricular ejection; LVOT, left ventricular outflow tract; NYHA, New York Heart Association; VLPs, ventricular late potentials. 
demonstrated that VLPs may not be a reliable indicator to detect myocardial fibrosis as detected by CMR in patients with HCM (24). Wada et al. reported that there was no significant difference in the presence of VLPs among the three groups classified by the level of myocardial fibrosis in patients with HCM (10). In the present study, LGE did not differ significantly between the two groups. Myocardial fibrosis in patients with HCM was qualitatively assessed in our study, and the results reflected the associations between VLPs and myocardial fibrosis from a macroscopic perspective. In summary, VLPs may not be a reliable indicator of myocardial fibrosis in patients with HCM, although the underlying mechanisms remain unclear. A possible cause of inconsistency between LGE and VLPs is the distribution and level of myocardial fibrosis. Left ventricular geometry may also contribute partly to the difference.

Positive VLPs can identify lethal cardiac events in patients with cardiac and extracardiac diseases, such as coronary heart disease, cardiomyopathy, and pulmonary sarcoidosis $(10,12,25,26)$. A previous study demonstrated the value of VLPs in the risk stratification of patients with dilated cardiomyopathy (13). In addition, a Japanese study reported that in HCM patients with positive VLPs, tissue fibrosis led to a greater risk of ventricular tachycardia/ventricular fibrillation at a mean follow-up duration of $5 \pm 4$ years (10).

In the present study, we found that VLPs were an independent risk factor of adverse events in patients with OHCM undergoing surgical septal myectomy. The primary end point of the study was a composite of clinical events. All-cause mortality, congestive heart failure requiring hospitalization, and new-onset stroke were lifethreatening or seriously influenced quality of the patients' life. Unexplained syncope is a risk factor for sudden cardiac death in HCM (27). The cause for unknown syncope in patients with HCM is not completely specified. As the myocardium in HCM presents with an underlying electrical instability from myocyte disarray and fibrosis, it is plausible that this mechanism increases the risk for adverse events including syncope (28).

Impaired cardiac function at admission was also a risk factor for poor prognosis in patients with OHCM in this study. NYHA classification is an indicator of preoperative cardiac function. Previous studies related to other cardiac surgeries have reported that patients with poor cardiac function at admission are often readmitted to the intensive care unit for serious cardiovascular complications (29). This finding suggests that patients with poor cardiac function at admission should be monitored closely during follow-up.

Preoperative VLPs might not be reliable as a gold standard risk factor for surgical outcomes in clinical practice. However, based on our study, we believe that identifying preoperative VLPs could be beneficial in clinical practice. VLPs indicates instability of electrical activity, which is associated with both fatal and nonfatal cardiac events. Preoperative examination to identify VLPs might help clinicians in making therapeutic decisions about the management of patients after the procedure. Moreover, patients with positive VLPs might have poorer outcomes and require closer clinical, echocardiographic, and electrocardiographic follow-up after septal myectomy.

The present study has some limitations. First, it was a single-center study in China, which led to small sample size. Second, the follow-up time was short, resulting in the detection of only a small number of adverse events in the study participants. This could affect the statistical robustness and generalizability of the results. Future investigators should expand the sample size and prolonged observation period to confirm these results. Finally, this was an observational study and had inherent disadvantages.

\section{Conclusions}

In this study, the presence of VLPs was an independent risk factor for adverse events in patients with OHCM after septal myectomy. This study included the largest number of patients with OHCM, to date, to investigate the clinical application value of VLPs. These results suggest that VLPs can be used as a risk factor for stratification.

\section{Acknowledgments}

We would like to thank Editage (www.editage.com) for English language editing.

Funding: This work was supported by grants from the National Key Research and Development Program of China (No. 2016YFC1300901).

\section{Footnote}

Reporting Checklist: The authors have completed the STROBE reporting checklist. Available at http://dx.doi. org/10.21037/apm-21-367

Data Sharing Statement: Available at http://dx.doi. org/10.21037/apm-21-367 
Conflicts of Interest: All authors have completed the ICMJE uniform disclosure form (available at http://dx.doi. org/10.21037/apm-21-367). The authors have no conflicts of interest to declare.

Ethical Statement: The authors are accountable for all aspects of the work in ensuring that questions related to the accuracy or integrity of any part of the work are appropriately investigated and resolved. The study conformed to the provisions of the Declaration of Helsinki (as revised in 2013). The study was approved by the Ethics Committee of Fuwai Hospital (NO. 2017-887) and informed consent was taken from all individual participants.

Open Access Statement: This is an Open Access article distributed in accordance with the Creative Commons Attribution-NonCommercial-NoDerivs 4.0 International License (CC BY-NC-ND 4.0), which permits the noncommercial replication and distribution of the article with the strict proviso that no changes or edits are made and the original work is properly cited (including links to both the formal publication through the relevant DOI and the license). See: https://creativecommons.org/licenses/by-nc-nd/4.0/.

\section{References}

1. Semsarian C, Ingles J, Maron MS, et al. New perspectives on the prevalence of hypertrophic cardiomyopathy. J Am Coll Cardiol 2015;65:1249-54.

2. Elliott PM, Anastasakis A, Borger MA, et al. 2014 ESC Guidelines on diagnosis and management of hypertrophic cardiomyopathy: the Task Force for the Diagnosis and Management of Hypertrophic Cardiomyopathy of the European Society of Cardiology (ESC). Eur Heart J 2014;35:2733-79.

3. Ommen SR, Maron BJ, Olivotto I, et al. Long-term effects of surgical septal myectomy on survival in patients with obstructive hypertrophic cardiomyopathy. J Am Coll Cardiol 2005;46:470-6.

4. Smedira NG, Lytle BW, Lever HM, et al. Current Effectiveness and Risks of Isolated Septal Myectomy for Hypertrophic Obstructive Cardiomyopathy. The Annals of Thoracic Surgery 2008;85:127-33.

5. Song C, Wang S, Guo Y, et al. Preoperative NT-proBNP Predicts Midterm Outcome After Septal Myectomy. J Am Heart Assoc 2019;8:e011075.

6. Woo A, Williams WG, Choi R, et al. Clinical and echocardiographic determinants of long-term survival after surgical myectomy in obstructive hypertrophic cardiomyopathy. Circulation 2005;111:2033-41.

7. Santangeli P, Infusino F, Sgueglia GA, et al. Ventricular late potentials: a critical overview and current applications. J Electrocardiol 2008;41:318-24.

8. Cripps TR, Counihan PJ, Frenneaux MP, et al. Signal-averaged electrocardiography in hypertrophic cardiomyopathy. J Am Coll Cardiol 1990;15:956-61.

9. Marcus FI, McKenna WJ, Sherrill D, et al. Diagnosis of arrhythmogenic right ventricular cardiomyopathy/ dysplasia: proposed modification of the Task Force Criteria. Eur Heart J 2010;31:806-14.

10. Wada Y, Aiba T, Matsuyama TA, et al. Clinical and Pathological Impact of Tissue Fibrosis on Lethal Arrhythmic Events in Hypertrophic Cardiomyopathy Patients With Impaired Systolic Function. Circ J 2015;79:1733-41.

11. Uzna ska-Loch B, Wiklo K, Trzos E, et al. Advanced and traditional electrocardiographic risk factors in pulmonary arterial hypertension: the significance of ventricular late potentials. Kardiol Pol 2018;76:586-93.

12. Yodogawa K, Seino Y, Ohara T, et al. Prognostic significance of ventricular late potentials in patients with pulmonary sarcoidosis. Heart Rhythm 2018;15:798-802.

13. Vester EG, Emschermann C, Stobbe U, et al. Late potentials and heart rate variability in heart muscle disease. Eur Heart J 1994;15 Suppl C:25-33.

14. Breithardt G, Cain ME, El-Sherif N, et al. Standards for analysis of ventricular late potentials using high-resolution or signal-averaged electrocardiography: a statement by a task force committee of the European Society of Cardiology, the American Heart Association, and the American College of Cardiology. J Am Coll Cardiol 1991;17:999-1006.

15. Mitchell C, Rahko PS, Blauwet LA, et al. Guidelines for Performing a Comprehensive Transthoracic Echocardiographic Examination in Adults: Recommendations from the American Society of Echocardiography. J Am Soc Echocardiogr 2019;32:1-64.

16. Zinman B, Wanner C, Lachin JM, et al. Empagliflozin, Cardiovascular Outcomes, and Mortality in Type 2 Diabetes. N Engl J Med 2015;373: 2117-28.

17. Sacco RL, Kasner SE, Broderick JP, et al. An updated definition of stroke for the 21 st century: a statement for healthcare professionals from the American Heart Association/American Stroke Association. Stroke 2013;44:2064-89.

18. Strickberger SA, Benson DW, Biaggioni I, et al. AHA/ 
ACCF Scientific Statement on the evaluation of syncope: from the American Heart Association Councils on Clinical Cardiology, Cardiovascular Nursing, Cardiovascular Disease in the Young, and Stroke, and the Quality of Care and Outcomes Research Interdisciplinary Working Group; and the American College of Cardiology Foundation: in collaboration with the Heart Rhythm Society: endorsed by the American Autonomic Society. Circulation 2006;113:316-27.

19. Lalani AP, Kanna B, John J, et al. Abnormal signalaveraged electrocardiogram (SAECG) in obesity. Obes Res 2000;8:20-8.

20. Huebner T, Goernig M, Schuepbach M, et al. Electrocardiologic and related methods of noninvasive detection and risk stratification in myocardial ischemia: state of the art and perspectives. Ger Med Sci 2010;8:Doc27.

21. Breithardt G, Becker R, Seipel L, et al. Non-invasive detection of late potentials in man--a new marker for ventricular tachycardia. Eur Heart J 1981;2:1-11.

22. Simson MB. Use of signals in the terminal QRS complex to identify patients with ventricular tachycardia after myocardial infarction. Circulation 1981;64:235-42.

23. Marstrand P, Axelsson A, Thune JJ, et al. Late potentials and their correlation with ventricular structure in patients

Cite this article as: Zhang $\mathrm{Y}$, Song C, Zhu C, Zhang J, Zheng X, Lu J, Wang S, Huang X. Preoperative ventricular late potentials and the risk of adverse events in patients with obstructive hypertrophic cardiomyopathy undergoing septal myectomy. Ann Palliat Med 2021;10(7):7280-7288. doi: 10.21037/ apm-21-367 with ventricular arrhythmias. Pacing Clin Electrophysiol 2017;40:1466-71.

24. Matsuki A, Kawasaki T, Kawamata H, et al. Ventricular late potentials and myocardial fibrosis in hypertrophic cardiomyopathy. J Electrocardiol 2020;58:87-91.

25. Goedel-Meinen L, Hofmann M, Ryba S, et al. Prognostic value of an abnormal signal-averaged electrocardiogram in patients with nonischemic dilated cardiomyopathy. Am J Cardiol 2001;87:809-12, A9.

26. Steinberg JS, Regan A, Sciacca RR, et al. Predicting arrhythmic events after acute myocardial infarction using the signal-averaged electrocardiogram. Am J Cardiol 1992;69:13-21.

27. Spirito P, Autore C, Rapezzi C, et al. Syncope and risk of sudden death in hypertrophic cardiomyopathy. Circulation 2009;119:1703-10.

28. Maron BJ, Roberts WC. Quantitative analysis of cardiac muscle cell disorganization in the ventricular septum of patients with hypertrophic cardiomyopathy. Circulation 1979;59:689-706.

29. Li S, Tang BY, Zhang B, et al. Analysis of risk factors and establishment of a risk prediction model for cardiothoracic surgical intensive care unit readmission after heart valve surgery in China: A single-center study. Heart Lung 2019;48:61-8. 